\title{
CERAMIC SURFACES AND SINTERING
}

\author{
Final Report \\ for Period July 1, 1987 to June 30, 1991 \\ Professor Laurence D. Marks, Principal Investigator \\ Professor D. Lynn Johnson
}

NORTHWESTERN UNIVERSITY

Evanston, Illinois 60208

August 1991

Prepared for

THE U. S. DEPARTMENT OF ENERGY

AGREEMENT NO. DE-FGO2-87ER45309

\section{DISCLAIMER}

This report was prepared as an account of work sponsored by an agency of the United States Government. Neither the United States Government nor any agency thereof, nor any of their employees, makes any warranty, express or implied, or assumes any legal liability or responsibility for the accuracy, completeness, or usefulness of any information, apparatus, product, or process disclosed, or represents that its use would not infringe privately owned rights. Reference herein to any specific commercial product, process, or service by trade name, trademark, manufacturer, or otherwise does not necessarily constitute or imply its endorsement, recommendation, or favoring by the United States Government or any agency thereof. The views and opinions of authors expressed herein do not necessarily state or reflect those of the United States Government or any agency thereof. 


\begin{abstract}
We produced uitrafine faceted alumina particles (UFPs), 20-50 $\mathrm{nm}$ in diameter, by an arcdischarge method and sintered small clusters of them as they were carried through a tube furnace by flowing gas. The experiment was conducted under near UHV clean environ.nent and specimens were examined in a UHV H9000 TEM. The UFPs produced in this study were transition alumina phases $(\gamma, \delta$ and $\theta)$. We found new $\delta$ and $\theta$ phases which were different from those in earlier studies. We developed two compuier programs to simulate the shape, 3-D arrangement, and random contact of UFPs. Gaps were discovered to exist between particles due to random contact and possibly rotation and slip after contact. As no shrinkage was found, surface diffusion was identified as the dominant mechanism that was responsible for the formation of "necks" by filling in the gaps. These necks had well-defined, atomically-sharp contact angles, which suggest that the neck growth process was controlled by faceting.
\end{abstract}




\section{INTRODUCTION}

Ultrafine particles have some unique characteristics which are different from larger particles. Traditional initial-stage sintering models assume a spherical shape and isotropic surface energy and diffusion coefficient. While these assumptions may be appropriate under special conditions with relatively large particles, the models break down in the sub-micrometer particle size ranges typically encountered in sinterable ceramic powders. The purpose of this research was to move beyond the traditional models in a study of the sintering behavior of alumina UFPs.

The progress report for the previous year described the construction of the equipment, i.e., the rising gas furnace, particle production apparatus, and interface to the UHV microscope which represented a substantial challenge. In this report we will concentrate more on the results, namely, (1) phases, structure, size distribution, and the shape of alumina UFPs; (2) computer simulation of random contact of UFPs; (3) mechanism of the sintering of alumina UFPs; (4) other structure and phase transformation.

\section{EXPERIMENTAL}

Alumina UFPs were produced by the arc-discharge gas-evaporation method. We developed a system, smaller in scale than similar setups of others, to produce just enough UFPs for clear observation in the microscope. Figure 1 shows a schematic diagram of the final arcdischarge setup, which was installed on the $150 \mathrm{~mm}$ bottom flange of the electrode chamber. Two aluminum electrodes (a wire anode $2 \mathrm{~mm}$ in diameter and a plate cathode $2 \mathrm{~mm}$ thick) were held in place by copper holders, and the arcing was generated inside an alumina retaining ring that sat on the plate electrode and helped stabilize the arc. A DC arc with a voltage drop of about $60 \mathrm{~V}$, and a current of $0.87 \mathrm{~A}$ was used.

The sintering furnace (Fig. 2), which is the heart of this project, consists of a vertical tube furnace, furnace chamber, electrode chamber (where the UFPs were generated), specimen chamber (where the sintered UFPs were collected), transfer rod, and UHV line. The resistance heating (Mo wire) tube furnace, with Mo foil shields, could reach $1500^{\circ} \mathrm{C}$. The furnace chamber served as a physical supporter for the furnace, and had several feedthroughs for cooling water lines, furnace power leads, forming gas (to protect the Mo from oxidation), thermocouples, pumping line, and viewport. The transfer rod connected to the specimen chamber placed the microscopic grid at the collection position just above the furnace and extracted it after collection. The system was equipped with a turbomolecular pump and an ion pump; after a thorough bakeout it could reach near UHV $\left(5 \times 10^{-7} \mathrm{~Pa}\right)$.

The preliminary bakeout, which cleaned up the system, was critical and could take over a week.

Before the arcing, the furnace was adjusted to be stable at the desired temperature. The UFPs emerged from the arc as a smoke, and were carried by the rising mixed gas $\left(20 \% \mathrm{O}_{2} / \mathrm{Ar}\right)$ into the tube furnace, where they sintered in pairs or chains. The particles were collected onto a microscopic grid on the specimen cartridge inside the specimen chamber. The transfer rod, with the grid, could be sealed, detached from the furnace system, and carried to and connected to the UHV-H9000 microscope while maintaining the high vacuum conditions. 


\section{RESULTS AND DISCUSSION}

\section{(1) Phases, structure, size distribution, and shape of alumina UFPs}

The particles produced were found to possess the basic $\gamma$-phase spinel structure, but could be more narrowly defined as $\gamma$ (cubic), $\delta$ (orthorhombic), and $\theta$ (monoclinic). A few electron diffraction patterns revealed a "new" form of the $\delta$ phase with lattice parameters of a $=1.58$, $\mathrm{b}=1.17$, and $\mathrm{c}=0.79 \mathrm{~nm}$, and space group $\mathrm{P} 2{ }_{1} 2_{1} 2_{1}$. The $\theta$ phase possibly was $\mathrm{a}=1.09 \mathrm{~nm}$, $\mathrm{b}=0.56 \mathrm{~nm}, \mathrm{c}=0.27 \mathrm{~nm}, \beta=103.3^{\circ}$, and space group $\mathrm{B} 2 / \mathrm{m}$. X-ray diffraction showed that the particles collected from the smoke were predominantly $\delta$ and $\gamma$ phases, those from the wall of the retaining ring were $\delta, \theta$ and $\alpha$ phases, and those from the plate were predominant $\alpha$ with smaller amount $\theta$ and $\delta$ phases.

Parallel electron energy loss spectroscopy (PEELS) was used to investigate the chemical nature of the sintered specimens. The results indicate there were no substantial metallic impurities contained within the particles. Thus, the formation and sintering processes were essentially contamination-free. However, PEELS is not sensitive enough to detect surface contamination.

Most particles were in the size range $20-50 \mathrm{~nm}$. Figure 3 shows particle size distribution diagrams of several runs. The results were not from a complete particle analysis, instead, they were measured directly from the TEM micrographs, and thus represented only a fraction of those particles collected on the grid. Figure 4 shows that the average size increased with increased gas pressure.

The particles were predominantly of the cuboctahedron shape (see Figure 5) with $\{110\}$ faces, with the latter decomposed into small $\{111\}$ hill-and-valley facets (see Figure 6). This is in spite of the fact that three phases were found. The shapes were similar because the three phases all are based on the cubic spinel crystal structure, the principal differences being the distribution of the cations, which causes small distortions of the lattice.

To facilitate the shape analysis of the particles, a computer simulation program, "3D", was written (in Turbo Pascal, more than seven thousand lines of source code). This program can show up to ten particles with designated sizes, orientation and position on the computer monitor. The program is interactive: one can give commands and see the result on the screen immediately. Because the micrographs were the projection images of the particles, "3D" helped us to learn the three dimensional geometric relationships among the particles. This was done by adjusting the parameters to obtain the best fit between the simulated profiles and those obtained in the microscope.

The decomposed $\{110\}$ faces cannot be predicted by the Wuiff construction. Nevertheless, if we ignore edge and corner effects, a simple minimum surface free energy condition can account for the sizes of these $\{110\}$ fures. Since the $\{110\}$ faces are composed of small $\{111\}$ facets, a change in the size of the $\{110\}$ faces produces a corresponding change in the total area of $\{111\}$, as well as $\{100\}$, faces. For a fixed shape, assumed to be the equilibrium shape, the ratio of $\gamma_{111} / \gamma_{100}$ is fixed. The size of the $\{110\}$ face was determined by finding the minimum total surface free energy of the particle as a function of $\{110\}$ area at constant particle volume. Table 1 shows the relative total surface energy of each shape with varying $\gamma_{111} / \gamma_{100}$ ratio and $\{110\}$ area, normalized to the value when $\gamma_{111} / \gamma_{100}=1$ and $V=0.40$. Here $V$ is a measure of the size of the $\{110\}$ faces on the cuboctahedron, ranging from zero (no $\{110\}$ ) to 0.5 (maximum $\{110\}$ ), given by 


$$
V=\frac{1}{2}\left(1-\frac{L}{L_{m}}\right)
$$

where $\mathrm{L}$ is the length of $(100)(1 \overline{11})$ intersection edge and $\mathrm{L}_{\mathrm{m}}$ is the maximum value if there are no $\{110\}$ faces. The $\gamma_{111} / \gamma_{100}$ ratio was

determined to be about one from the micrographs, so $\mathrm{V}=0.4$ should be the equilibrium shape. Comparisons between micrographs and the "3D" simulations indicated that $\gamma_{111} / \gamma_{100} \approx 1$ and $\mathrm{V}$ ranged from 0.35 to 0.4 , in satisfactory agreement with this calculation.

One would expect the edges and corners to raise the total energy, and therefore restrain the decomposition of $\{110\}$ facets; this can explain the fact that the observed values of $\mathrm{V}$ are slightly lower than that of the calculation. For particles smaller than $20 \mathrm{~nm}$ the validity of this calculation is doubtful, since the edge and corner effect can no longer be ignored.

\section{(2) Computer simulation of random contact}

Alumina particles collected before sintering were in chains, and had no obvious "necks" between them. A variety of particle contacts were observed. In the absence of constraint from neighboring particles, the majority of contacts were face-to-face (with or without small angle wedge-shaped gaps) with nearly full overlap. While we were not able to observe all the collected particles, and could not determine precisely the fraction of face-to-face contacts, there appeared to be far too many to account for on the basis of random contact. In order to better quantify this, a computer program, "Bump", was written to simulate the random contact of two cuboctahedra with $\{110\}$ faces with specified size and shape parameters.

Two polyhedra with mathematically sharp corners and edges and planar faces that are brought into contact at random will not contact by corner-to-corner, corner-to-edge, edge-to-face or face-to-face. The only two possible solutions are corner-to-face and edge-to-edge. The smallest building block for alumina particles is the atom, which has a definite spherical size (say $0.2 \mathrm{~nm}$ ); Lhus, all six of the contacts described above become possible. For this reason, when a corner approached another element to within $0.2 \mathrm{~nm}$, we counted it as a corner contact. Similarly, when an edge and a face, or a face and another face, had an inter-angle smaller than $2^{\circ}$, we counted it a contact.

The first particle was positioned at the origin aligned with the axes. The second particle was positioned at random, with randomly chosen orientation, and moved toward the origin until contact was made. The contact was analyzed and assigned to one of the six categories. It was found that the shape parameters and the relative sizes of the two particles had small effects on the results.

The results for $\mathrm{V}=0.4$ and equal radius, after 10000 random contacts, were as follows:

(1) corner to corner

(2) comer to edge

(3) corner to face

(4) edge to edge

(5) edge to face

(6) face to face

$\begin{aligned} 11 & {[0.11 \%] } \\ 358 & {[3.58 \%] } \\ 3638 & {[36.38 \%] } \\ 3490 & {[34.90 \%] } \\ 2437 & {[24.37 \%] } \\ 66 & {[0.66 \%] }\end{aligned}$


This confirmed that the chances for face-to-face contact is small. Note that in this simulation a contact with an edge or face could take place anywhere within the boundaries of the edge or face, and a significant overhang, even on face-to-face contacts, is to be expected, though never ubserved with unconstrained particles. The large discrepancy between the simulated and observed types of contact leads to the conclusion that there must have been rotation and sliding of one particle with respect to the other after or just before contact was made. This would be driven by reduction of the total surface and interfacial free energy of the pair of particles.

\section{(3) Sintering mechanisms}

The sintered alumina UFPs pairs have very clear "necks." From sintering theories we know that several sintering mechanisms, i.e., volume, grain boundary, and surface diffusion and evaporation-condensation, can occur at same time. However, only the iirst two can create shrinkage, or approach of the particles towards each other; the second two move the surface atoms onto the neck surface without shrinkage. Comparing the "3D" simulation with microscope images showed no noticeable shrinkage. Moreover, of ten there were very clear gaps between particle pairs before sintering (Fig.7 (a)-(c)). Atoms moved from the particle surfaces to fill these gaps during sintering. The vapor pressure of alumina is sufficiently low at the sintering temperatures that evaporation-condensation should not be a major factor. Traditional sintering theory would predict surface diffusion to be much more important than grain boundary or volume diffusion for particles of this size range, and there is nothing in the sintering of polyhedral particles that would nullify this.

A simple calculation tells how large the fill-in volume of the gaps should be. Based on the typical shape of particles, radii, we calculated the gap volume for six different inter-angles of four types of contacts for five radii. The four contacts are: (1) two $\{100\}$ faces contact by one of their edges between $\{100\}$ and $\{111\}$ (this will be represented as $\{100\} /\{100\}$, edge100111), (2) $\{100\} /\{100\}$, edge100110, (3) $\{111\} /\{111\}$, edge100111, (4) $\{111\} /\{111\}$, edge111110. Table 2 lists the results of fill-in volume, volume percentage, and the equivalent thickness, the reduction in radius of the particles if the fill-in volume were removed from the entire surface of the particles. The most common inter-angle found in the micrographs was about 5 to 10 degrees, so from Table 2, we see that less than about $0.75 \%$ of the total volume of the two particles was needed to fill in the gap.

\section{(4) Other structures}

Twins and stacking faults occurred frequently in the sintered alumina UFPs. There is little strain induced by contact stresses, contrary to the metal UFP case, and internal faulting was found instead. This is probably because the ceramic UFPs cannot plastically deform easily like metals. A few particles appeared to be multiply-twinned particles (MTPs), such as in Fig.8.

In some rare cases where particles were aligned perfectly, the grain (or perhaps phase) boundary migrated out of the neck into one of the particles. Unfortunately, it is difficult to determine the phase of a single particle because the electron beam was too large and the charging effect induced by focusing may eject the particle. 


\section{CONCLUSION}

The key of this research was the UHV-H9000, the first true UHV high resolution TEM in the world, which made this project possible. We used the arc-discharge method to produce alumina UFPs $(20-50 \mathrm{~nm})$, which were sintered and collected in a very clean environment. The alumina UFPs were transition aluminas, based on the spinel structure, and exhibited cubic cuboctahedron shape with (decomposed) $\{110\}$ faces. This shape can be explained easily by a minimum surface free energy argument, and a calculation based on the Wulff construction demonstrated this result. The "necks" of sintered UFPs depended on their original contact conditions; atoms filled in the gaps between particles by surface diffusion. Two computer programs that simulated the 3-D relationship and the random contact of alumina UFPs were developed to explain the present results. 
Table 1. Relative total surface energy of cuboctahedron shapes of various $\gamma_{111} / \gamma_{100}$ ratio (GR) and $\{110\}$ area factor $(V)$. The minimum for each row is underlined.

\begin{tabular}{||r|rrrrrr||}
\hline \multicolumn{1}{|c|}{ GR } & \multicolumn{7}{|c|}{110} arca factor. V \\
\cline { 2 - 7 } 0.60 & 0.20 & 0.25 & 0.30 & 0.35 & 0.40 & 0.45 \\
\hline \hline 0.65 & 247.234 & 228.700 & 212.350 & 197.852 & 184.935 & 173.376 \\
0.70 & 20.0703 & 18.7097 & 17.5210 & 16.4774 & 15.5575 & 14.7437 \\
0.75 & 6.3698 & 5.9877 & 5.6561 & 5.3673 & 5.1153 & 4.8952 \\
0.80 & 3.1552 & 2.9932 & 2.8533 & 2.7322 & 2.6276 & 2.5374 \\
0.85 & 2.0000 & 1.9152 & 1.8422 & 1.7794 & 1.7258 & 1.6805 \\
0.90 & 1.4859 & 1.4356 & 1.3925 & 1.3559 & 1.3253 & 1.3001 \\
0.95 & 1.2292 & 1.1973 & 1.1703 & 1.1480 & 1.1301 & 1.1164 \\
0.96 & 1.0956 & 1.0751 & 1.0584 & 1.0454 & 1.0360 & 1.0304 \\
0.97 & 1.0781 & 1.0594 & 1.0444 & 1.0329 & 1.0250 & 1.0209 \\
0.98 & 1.0630 & 1.0460 & 1.0325 & 1.0225 & 1.0161 & 1.0133 \\
0.99 & 1.0500 & 1.0347 & 1.0227 & 1.0141 & 1.0090 & 1.0077 \\
1.00 & 1.0390 & 1.0252 & 1.0147 & 1.0074 & 1.0037 & 1.0038 \\
1.01 & 1.0298 & 1.0175 & 1.0083 & 1.0024 & 1.00000 & 1.0015 \\
1.02 & 1.0223 & 1.0113 & 1.0035 & 0.9989 & 0.9978 & 1.0007 \\
1.03 & 1.0163 & 1.0067 & 1.0001 & 0.9968 & 0.9970 & 1.0013 \\
1.04 & 1.0084 & 1.0034 & 0.9981 & 0.9961 & 0.9976 & 1.0032 \\
1.05 & 1.0064 & 1.0006 & 0.9974 & 0.9966 & 0.9994 & 1.0065 \\
1.10 & 1.0127 & 1.0128 & 1.0160 & 1.0228 & 1.0338 & 1.0502 \\
1.15 & 1.0424 & 1.0483 & 1.0576 & 1.0711 & 1.0900 & 1.1156 \\
1.20 & 1.0927 & 1.1046 & 1.1205 & 1.1416 & 1.1695 & 1.2064 \\
1.25 & 1.1627 & 1.1810 & 1.2043 & 1.2343 & 1.2732 & 1.3242 \\
1.30 & 1.2533 & 1.2787 & 1.3105 & 1.3510 & 1.4036 & 1.4727 \\
1.35 & 1.3665 & 1.4000 & 1.4418 & 1.4954 & 1.5653 & 1.6582 \\
1.40 & 1.5060 & 1.5490 & 1.6031 & 1.6730 & 1.7654 & 1.8906 \\
1.45 & 1.6778 & 1.7321 & 1.8013 & 1.8923 & 2.0151 & 2.1856 \\
1.50 & 1.8906 & 1.9586 & 2.0472 & 2.1665 & 2.3318 & 2.5692 \\
1.55 & 2.1580 & 2.2434 & 2.3580 & 2.5168 & 2.7445 & 3.0870 \\
1.60 & 2.5021 & 2.6104 & 2.7613 & 2.9787 & 3.3049 & 3.8283 \\
& & & & & & \\
\hline
\end{tabular}




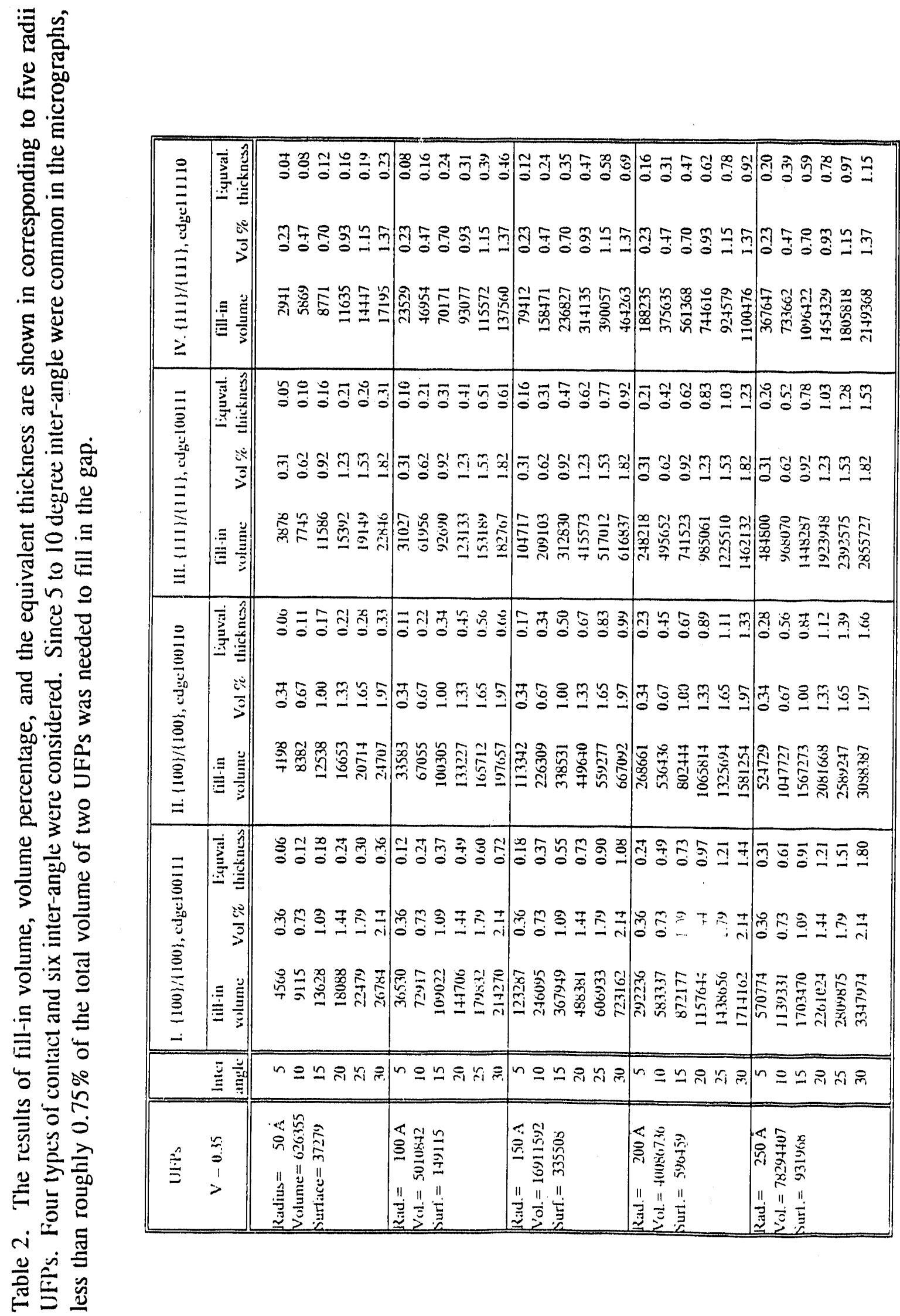




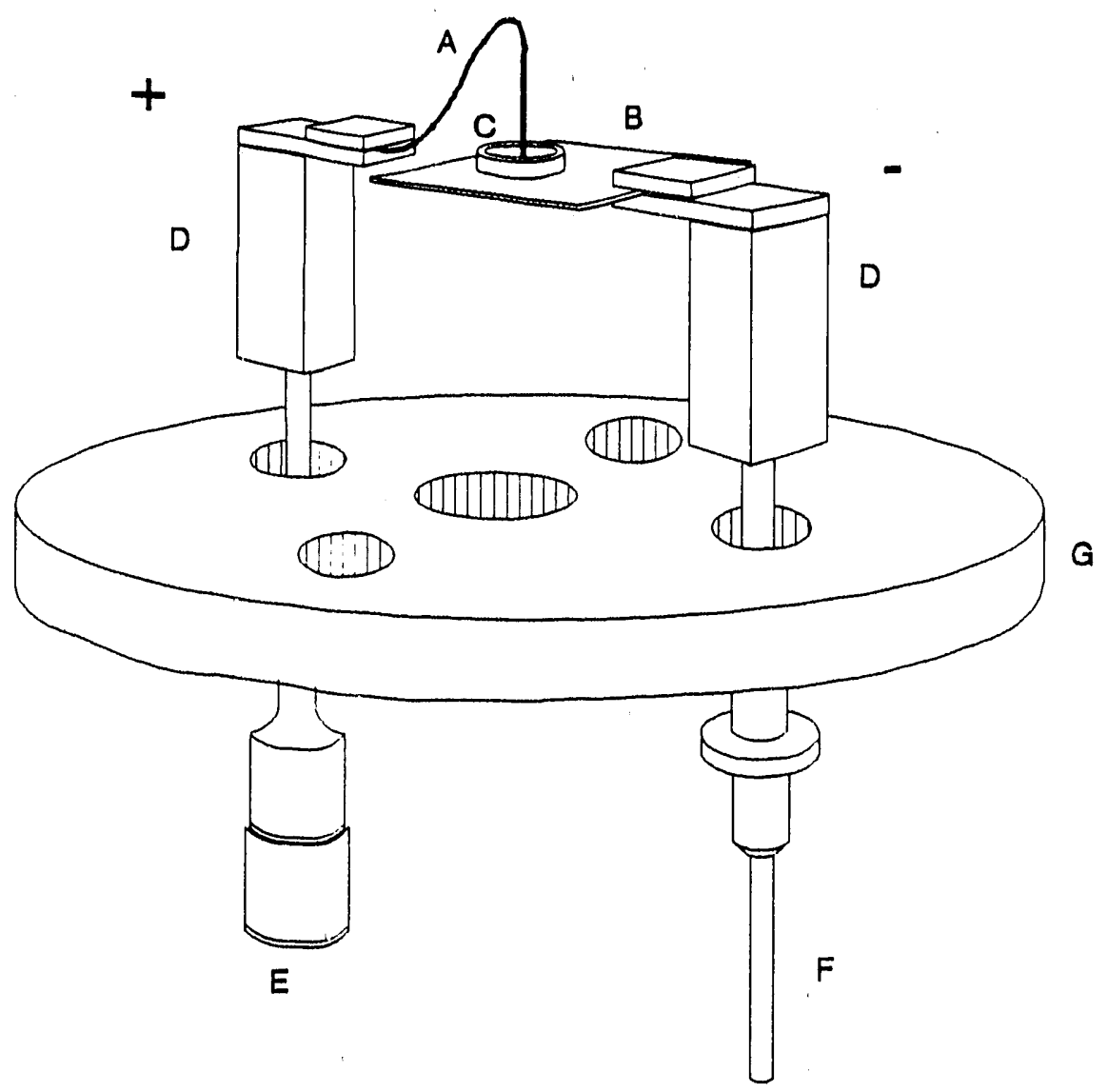

Figure 1. A schematic representation of the arc-discharge setup. The aluminum wire anode (A) and aluminum plate cathode (B) are held by copper holders (D), which are installed on the 150 $\mathrm{mm}$ bottom flange $(\mathrm{G})$ of the electrode chamber and connected to the outside by a rotary feedthrough for adjusting the arcing position $(E)$ and an electrode feedthrough $(F)$. The arcing is confined inside an alumina retaining ring (C) to keep the arc intensity stable. 


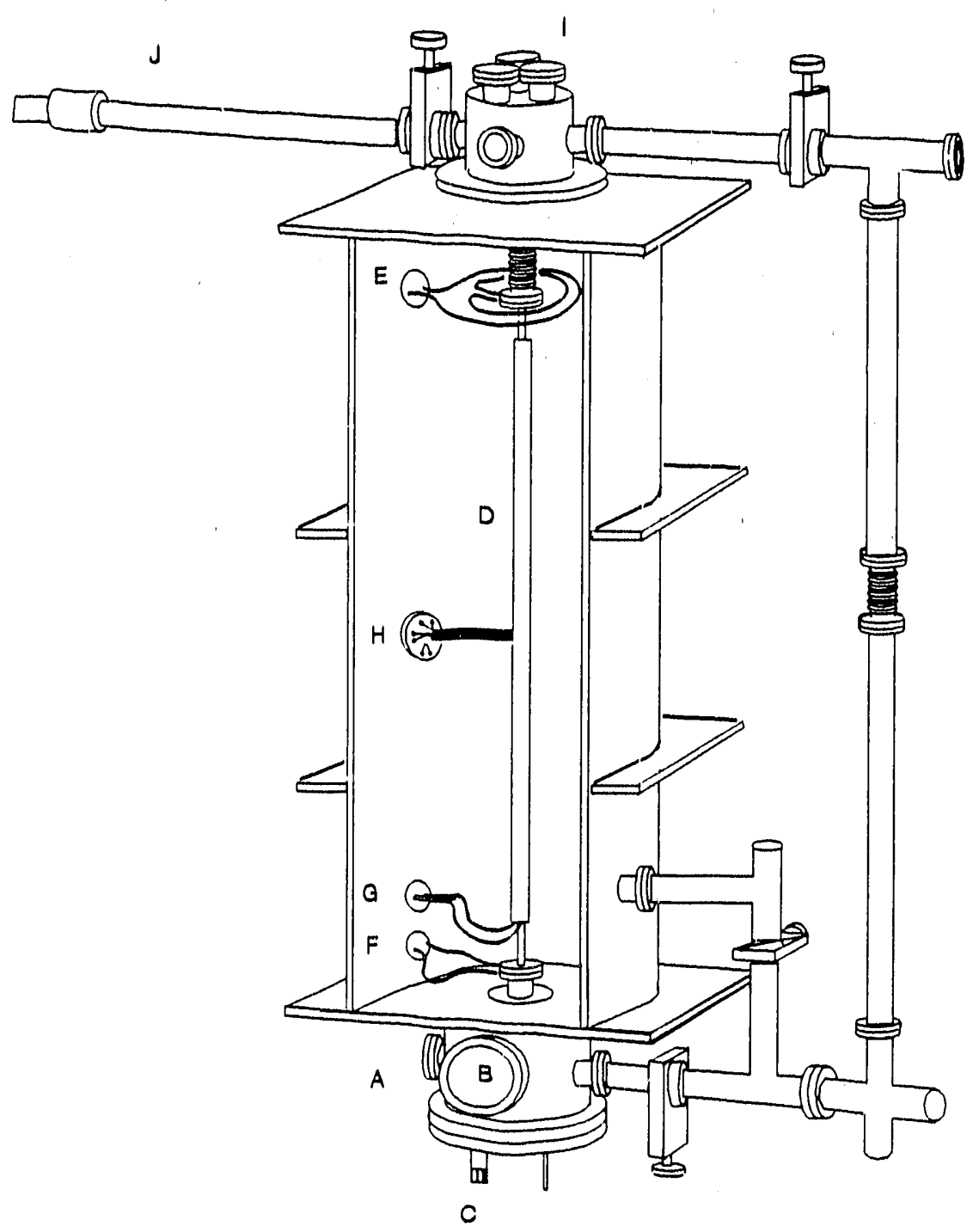

Figure 2. The sintering furnace (schematic, without the door). The arc-discharge setup (see Fig. 1) is installed in the electrode chamber (A), where a window (B) allows viewing of the arcing, with the rotary feedthrough $(C)$ to adjust the arc position. The UFP smoke, carried by $20 \% \mathrm{O}_{2} / \mathrm{Ar}$ gas, rises into the furnace (D) where the clusters of particles are sintered. The seals at the ends of the furnace are surrounded by cooling lines $(E$ and $F)$. Power is fed at $(G)$ and the furnace temperature is measured with four thermocouples $(\mathrm{H})$. Sintered particles are collected inside the specimen chamber (I) onto a grid, which is on a specimen cartridge held by a transfer rod contained within the transfer section $(\mathrm{J})$. 
Size Distribution of [ll rafine . Ilumina ( $\operatorname{arc} \# 58,13400 \mathrm{~Pa}_{1} 1600^{\circ} \mathrm{C}, 0.14 \mathrm{sec}$ )

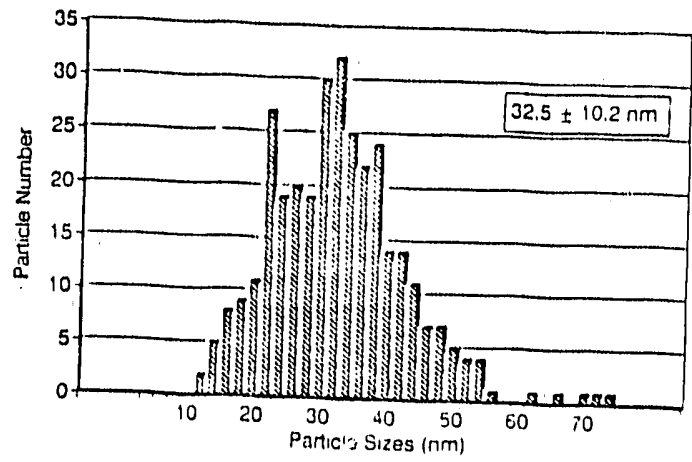

$\left(\operatorname{arc} \# 59,10650 \mathrm{~Pa}, 1500^{\circ} \mathrm{C}, 0.125 \mathrm{sec}\right)$

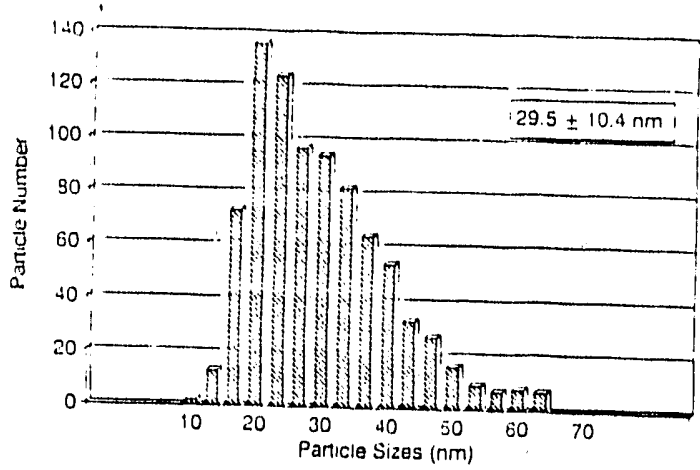

( $\operatorname{arc} \# 60,10700 \mathrm{~Pa}$, room $\mathrm{T}, 0.57 \mathrm{sec}$ )

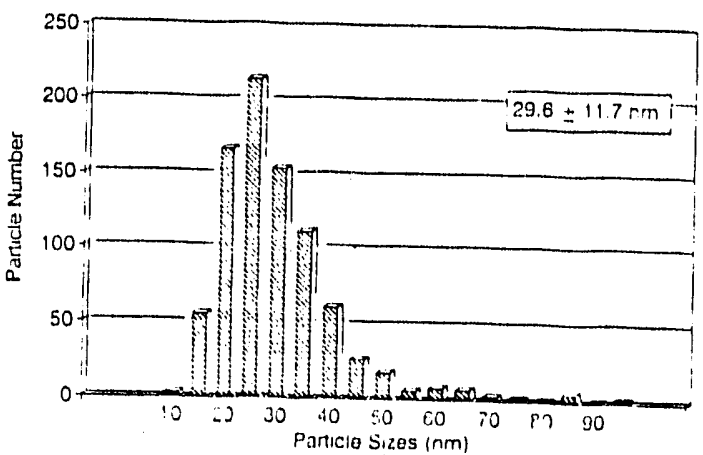

Figure 3. Particle size distribution diagrams for several runs. 


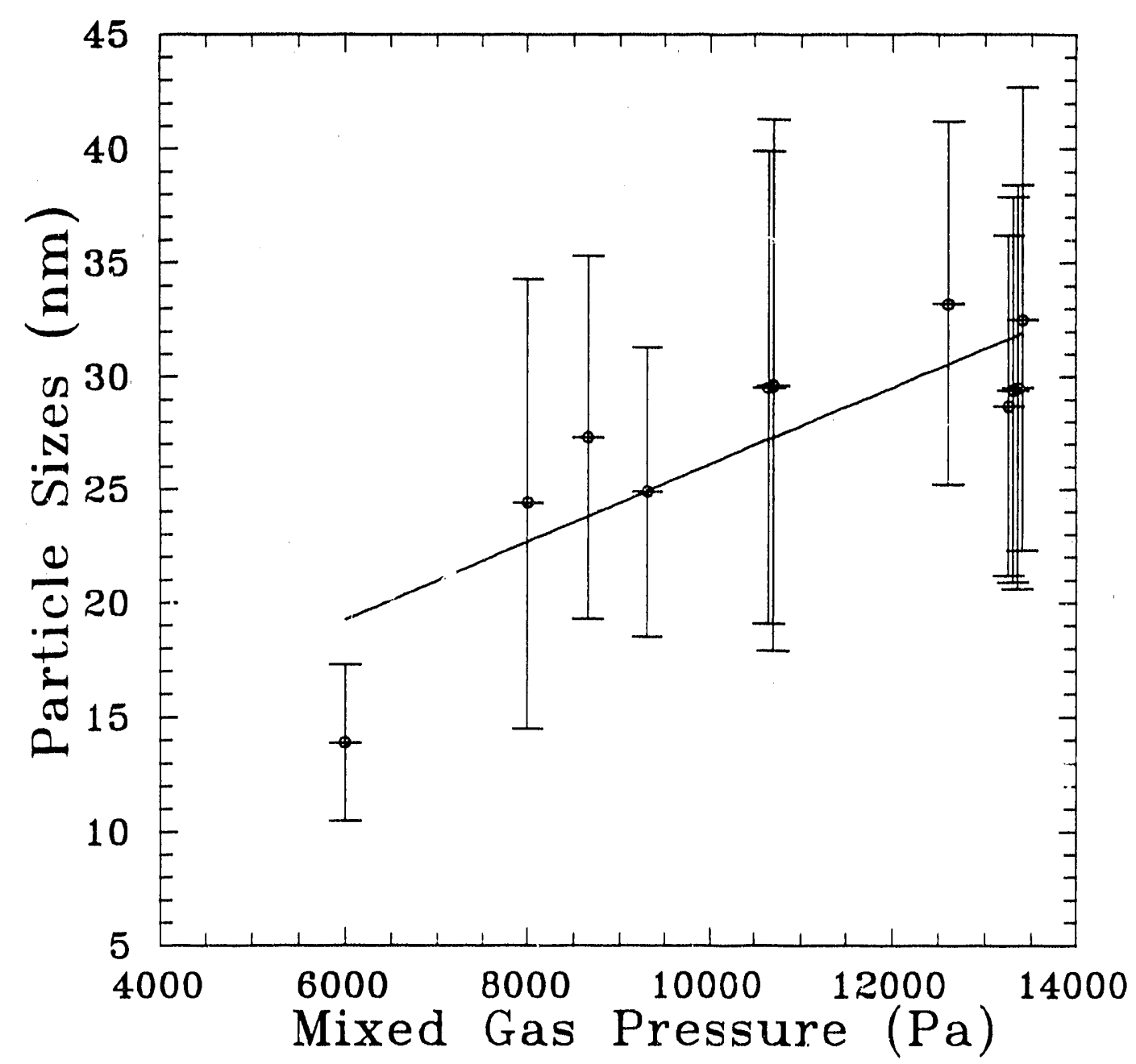

Figure 4. Particle size as a function of gas pressuie during arcing. 

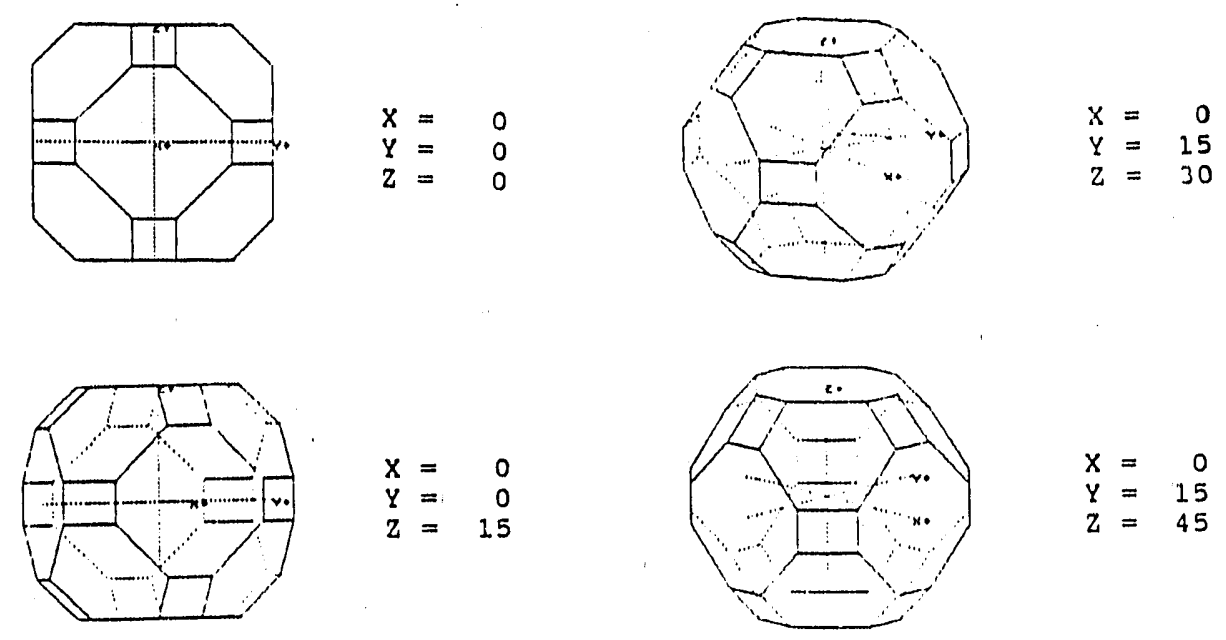

$X=0$
$y=0$
$Z=15$

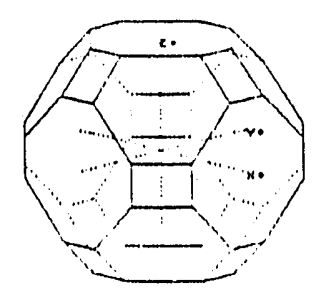

$x=0$
$y=15$
$z=45$

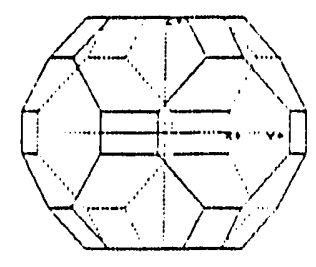

$X=0$
$Y=0$
$Z=30$

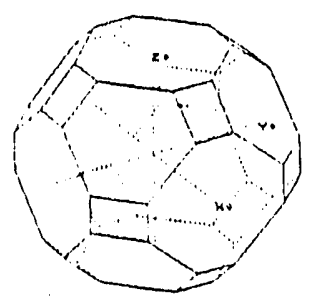

$x=0$
$y=30$
$z=30$

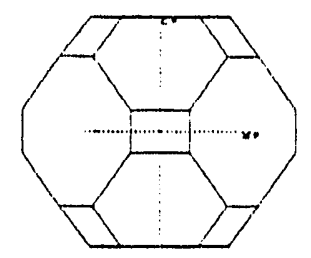

$x=0$
$y=0$
$Z=45$

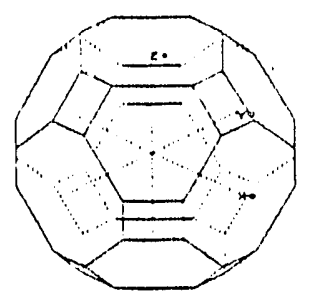

$X=0$
$Y=30$
$Z=45$
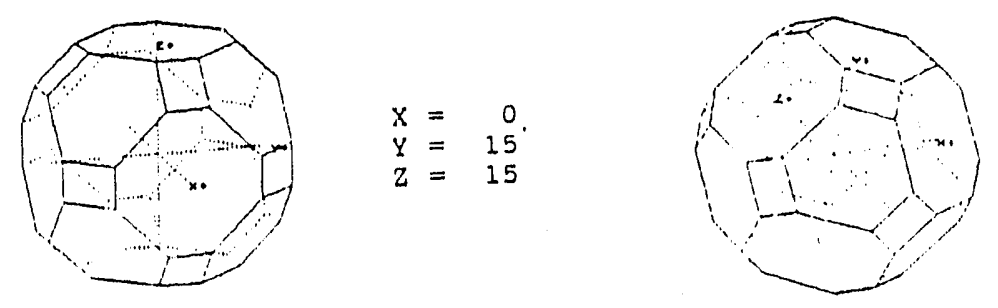

$X=45$
$Y=45$
$Z=45$

Figure 5. Ten views of a cuboctahedra with $\{110\}$ faces in various orientations. Each shape has 26 faces: $6\{100\}$ (octagon), $8\{111\}$ (hexagon), and $12\{110\}$ (rectangle). This figure was generited by the computer program "3D". 
(101)
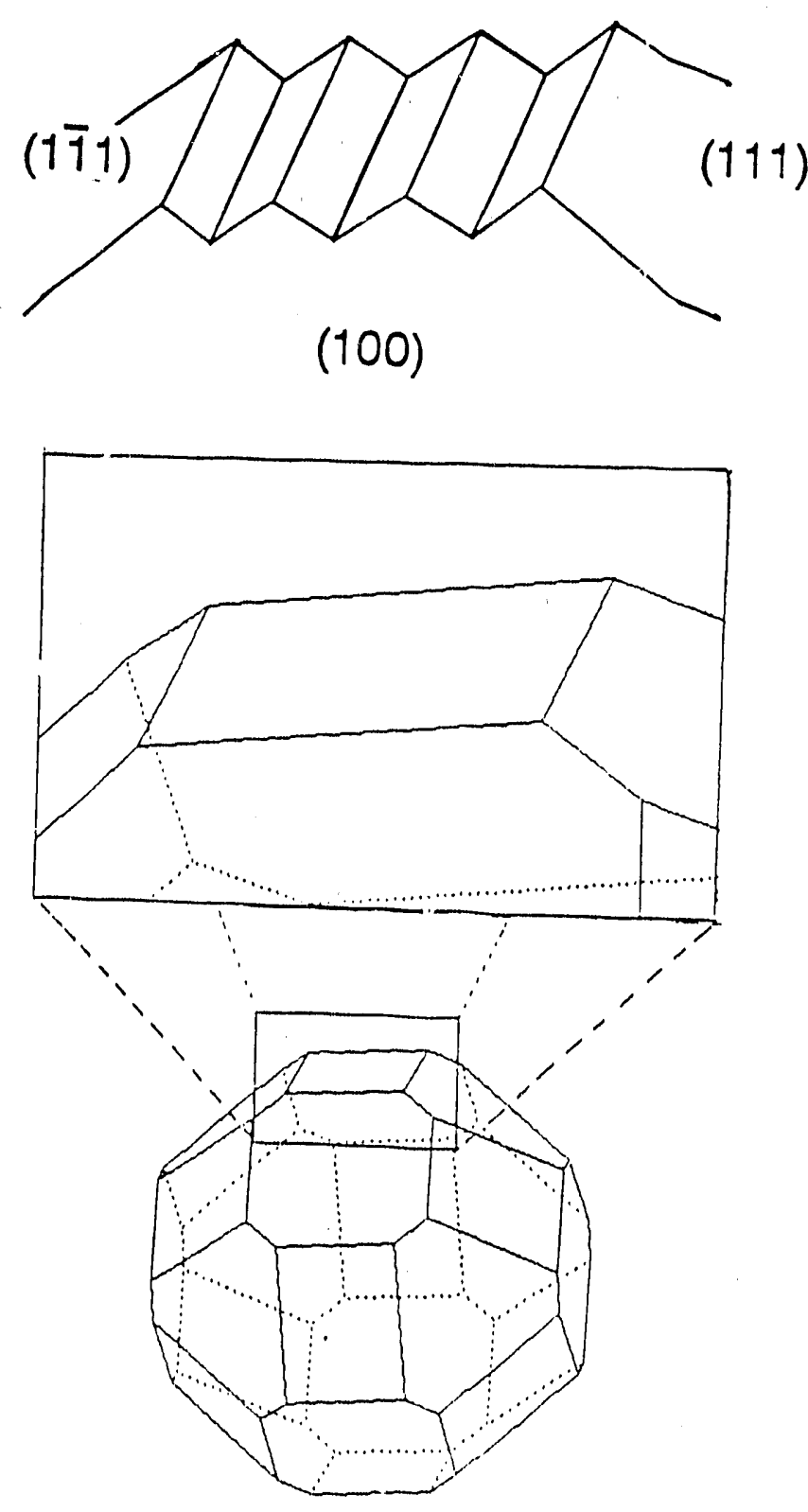

Figure 6 . The $\{110\}$ faces were actually decomposed into a hill-and-valley structure composed of small $\{111\}$ facets. The surface geometry of the enlarged rectangle face, (101), is shown above. 

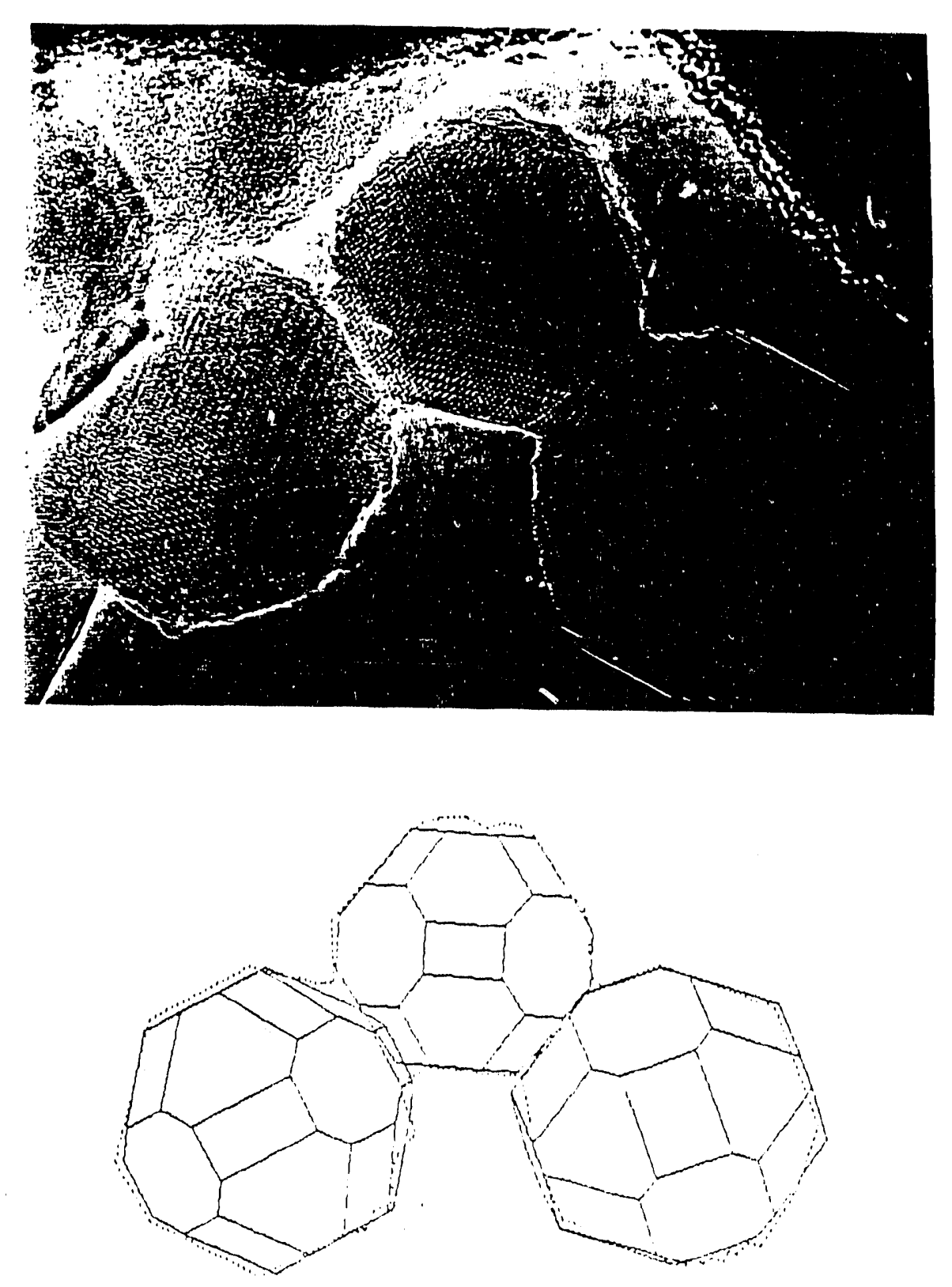

(a)

Figure 7 (a)-(c). Two or three sintered alumina UFPs with clear "gaps" between them which have been filled with atoms. The soild lines in the bottom figure were generated by the "ID" program, and the oroken lines. were araced from the micrograph. 

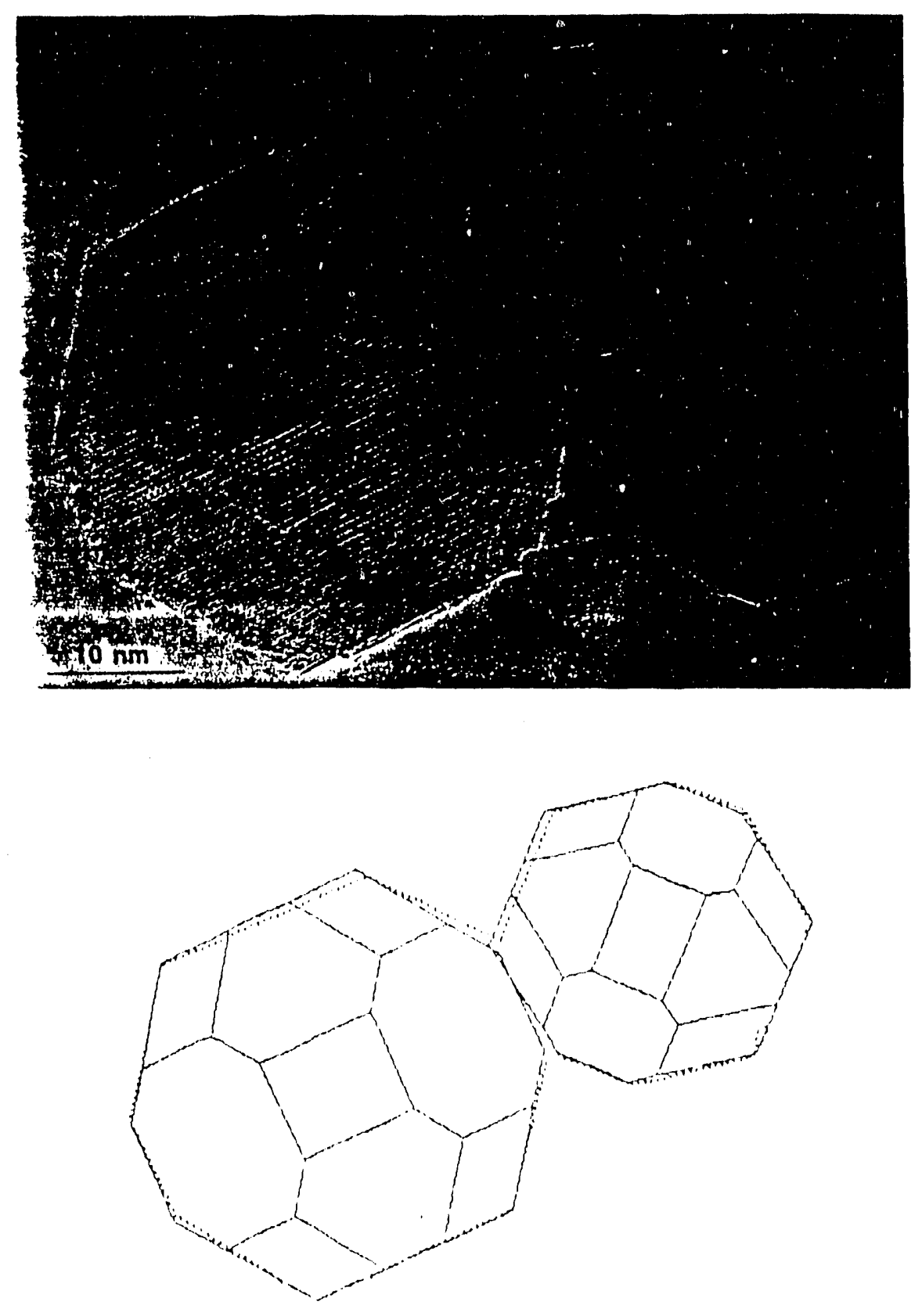

(b)

Figura (b). 

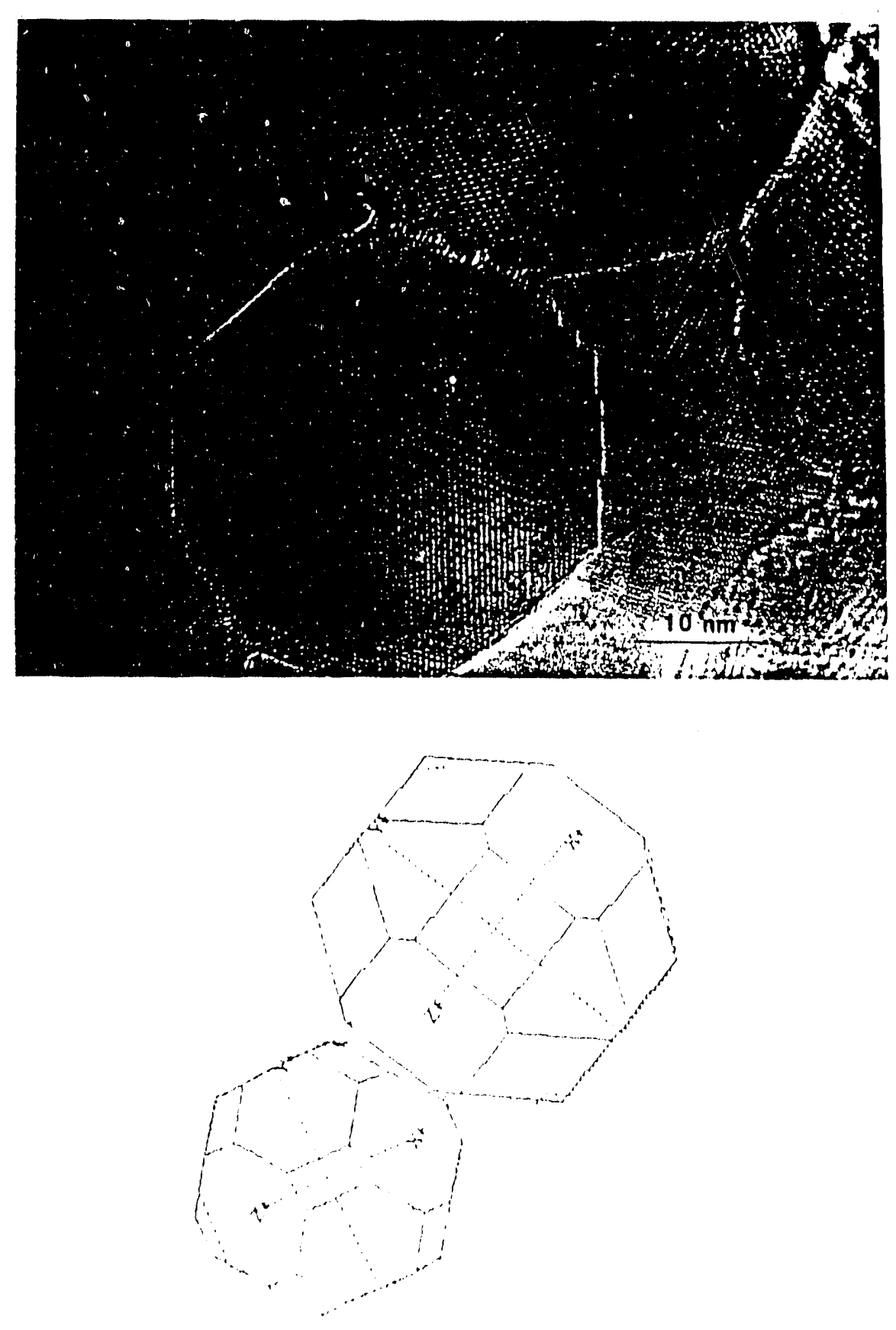

l゙!

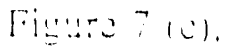




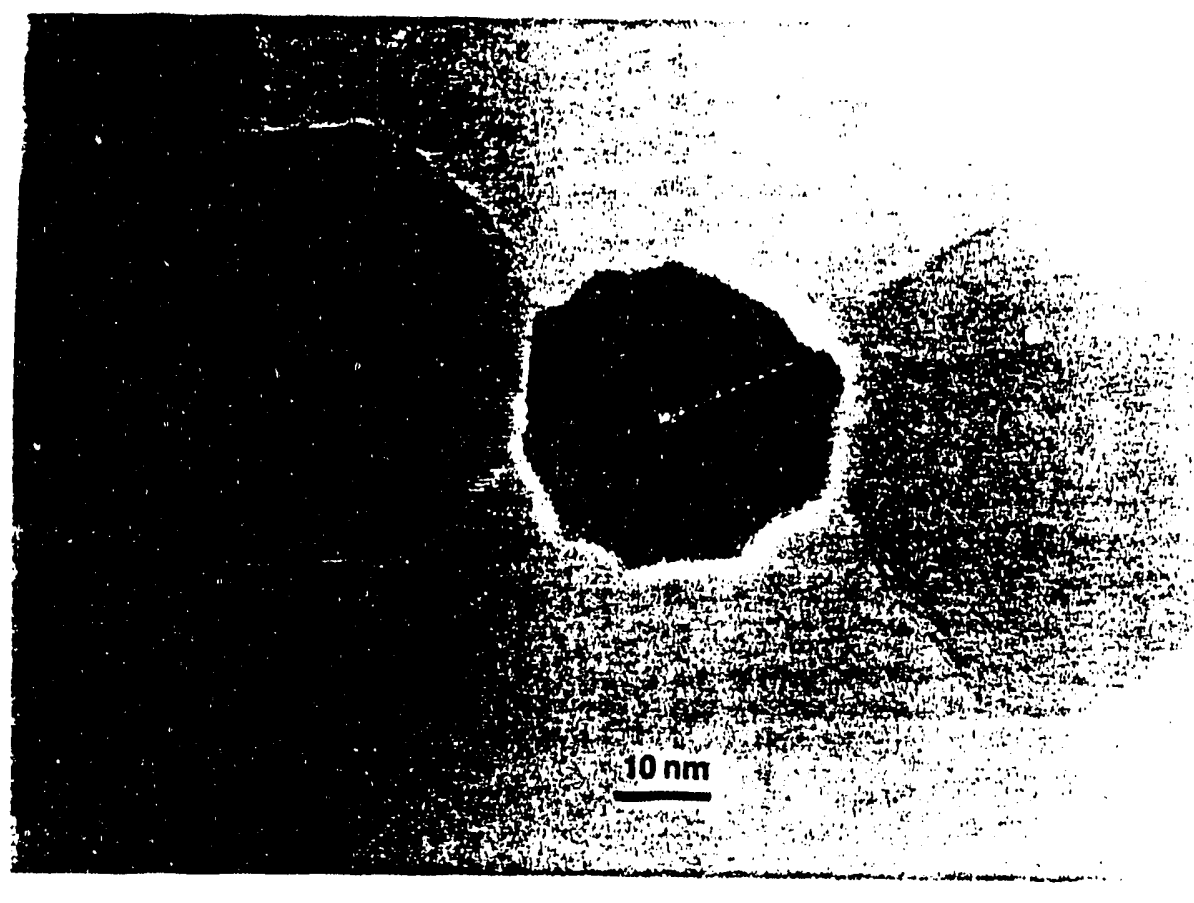

Figure 8. A possible multiple-twinned particle (MTP) of a UFP alumina. 

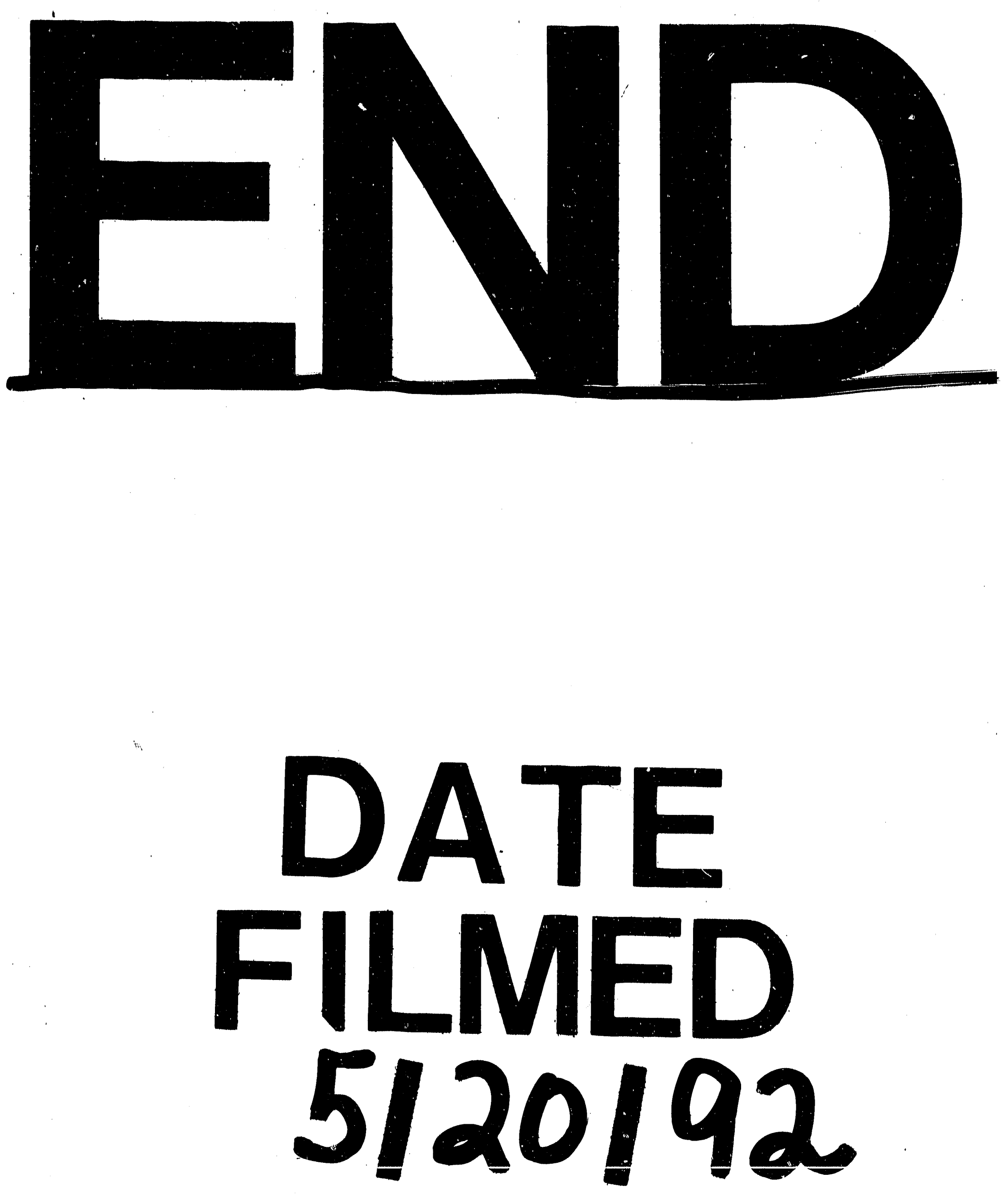
